\title{
The Focal Developable and the Binormal Indicatrix of a Nonlightlike Curve in Minkowski 3-Space
}

\author{
Donghe PEI and Takashi SANO \\ North East Normal University and Hokkaido University \\ (Communicated by M. Oka)
}

\begin{abstract}
We define the binormal indicatrix and focal developable of a nonlightlike curve in Minkowski 3space. We establish the relationships between singularities of these subjects and geometric invariants of curves under the action of Lorentzian group.
\end{abstract}

\section{Introduction.}

There are several articles concerning 'generic differential geometry' in the Euclidean space $[1-5,7,9,12$, etc.]. The main tools in these articles are the distance-squared functions and the height functions on submanifolds. In this paper we introduce the notion of Lorentzian height functions and Lorentzian distance-squared functions on timelike curves or spacelike curves in Minkowski 3-space. We also define the notion of binormal indicatrices and focal developables of timelike curves or spacelike curves in Minkowski 3-space, and establish the relationships between singularities of these subjects and geometric invariants of the curve under the action of Lorentzian group as applications of standard techniques of singularity theory for the above functions. For the basic notions in Lorentzian geometry, see [11].

Let $\mathbf{R}^{3}=\left\{\left(x_{1}, x_{2}, x_{3}\right) \mid x_{1}, x_{2}, x_{3} \in \mathbf{R}\right\}$ be a 3 -dimensional vector space, $\boldsymbol{x}=\left(x_{1}, x_{2}, x_{3}\right)$ and $\boldsymbol{y}=\left(y_{1}, y_{2}, y_{3}\right)$ be two vectors in $\mathbf{R}^{3}$, the pseudo scalar product of $\boldsymbol{x}$ and $\boldsymbol{y}$ is defined by $\langle\boldsymbol{x}, \boldsymbol{y}\rangle=-x_{1} y_{1}+x_{2} y_{2}+x_{3} y_{3} .\left(\mathbf{R}^{3},\langle\rangle,\right)$ is called a 3-dimensional pseudo Euclidean space, or Minkowski 3-space. We denote $\mathbf{R}_{1}^{3}$ as $\left(\mathbf{R}^{3},\langle\rangle,\right)$. For any $\boldsymbol{x}=\left(x_{1}, x_{2}, x_{3}\right), \boldsymbol{y}=\left(y_{1}, y_{2}, y_{3}\right) \in$ $\mathbf{R}_{1}^{3}$, the pseudo vector product of $\boldsymbol{x}$ and $\boldsymbol{y}$ is defined by

$$
\boldsymbol{x} \wedge \boldsymbol{y}=\left|\begin{array}{ccc}
-e_{1} & e_{2} & e_{3} \\
x_{1} & x_{2} & x_{3} \\
y_{1} & y_{2} & y_{3}
\end{array}\right|=\left(-\left(x_{2} y_{3}-x_{3} y_{2}\right), x_{3} y_{1}-x_{1} y_{3}, x_{1} y_{2}-x_{2} y_{1}\right) .
$$

Moreover, $\boldsymbol{x}$ in $\mathbf{R}_{1}{ }^{3}$ is called a spacelike vector, a lightlike vector or a timelike vector if $\langle\boldsymbol{x}, \boldsymbol{x}\rangle>0,\langle\boldsymbol{x}, \boldsymbol{x}\rangle=0$ or $\langle\boldsymbol{x}, \boldsymbol{x}\rangle<0$ respectively. For $\boldsymbol{x} \in \mathbf{R}_{1}^{3}$, the norm of $\boldsymbol{x}$ is defined by $\|x\|=\sqrt{\operatorname{sign}(\boldsymbol{x})\langle\boldsymbol{x}, \boldsymbol{x}\rangle}$, and $\boldsymbol{x}$ is called a unit vector if $\|x\|=1$, where $\operatorname{sign}(\boldsymbol{x})$ denotes the 
signature of $x$ which is given by

$$
\operatorname{sign}(x)= \begin{cases}1 & x: \text { spacelike } \\ 0 & x: \text { lightlike } \\ -1 & x: \text { timelike }\end{cases}
$$

Let $\gamma: I \rightarrow \mathbf{R}_{1}^{3} ; \gamma(t)=\left(x_{1}(t), x_{2}(t), x_{3}(t)\right)$ be a smooth regular curve in $\mathbf{R}_{1}^{3}$ (i.e., $\dot{\gamma}(t) \neq 0$ for any $t \in I$ ), where $I$ is an open interval. For any $t \in I$, the curve $\gamma$ is called a spacelike curve, a lightlike curve or a timelike curve if $\langle\dot{\gamma}(t), \dot{\gamma}(t)\rangle>0,\langle\dot{\gamma}(t), \dot{\gamma}(t)\rangle=0$, $\langle\dot{\gamma}(t), \dot{\gamma}(t)\rangle<0$ respectively. We call $\gamma$ a nonlightlike curve if $\gamma$ is a timelike curve or a spacelike curve. The arc-length of a nonlightlike curve $\gamma$, measured from $\gamma\left(t_{0}\right), t_{0} \in I$ is

$$
s(t)=\int_{t_{0}}^{t}\|\dot{\gamma}(t)\| d t .
$$

Then the parameter $s$ is determined such that $\left\|\gamma^{\prime}(s)\right\|=1$ for the nonlightlike curve, where $\gamma^{\prime}(s)=d \gamma / d s(s)$. So we say that a nonlightlike curve $\gamma$ is parameterized by arc-length if it satisfies that $\left\|\gamma^{\prime}(s)\right\|=1$. Throughout the reminder in this paper we denote the parameter $s$ of $\gamma$ as the arc-length parameter. Let us denote $t(s)=\gamma^{\prime}(s)$, and we call $t(s)$ a unit tangent vector of $\gamma$ at $s$. We define the curvature by $k(s)=\sqrt{\left|\left\langle\gamma^{\prime \prime}(s), \gamma^{\prime \prime}(s)\right\rangle\right|}$. If $k(s) \neq 0$ then the unit principal normal vector $\boldsymbol{n}(s)$ of the curve $\gamma$ at $s$ is given by $\gamma^{\prime \prime}(s)=k(s) \cdot \boldsymbol{n}(s)$. We denote that $\varepsilon(\gamma(s))=\operatorname{sign}(t(s))$ and $\delta(\gamma(s))=\operatorname{sign}(\boldsymbol{n}(s))$. The unit vector $\boldsymbol{b}(s)=\boldsymbol{t}(s) \wedge \boldsymbol{n}(s)$ is called a unit binormal vector of the curve $\gamma$ at $s$. Then the following Frenet-Serret type formula holds:

$$
\left\{\begin{array}{l}
t^{\prime}(s)=k(s) \cdot \boldsymbol{n}(s) \\
\boldsymbol{n}^{\prime}(s)=-\varepsilon(\gamma(s)) \cdot \delta(\gamma(s)) \cdot k(s) \cdot \boldsymbol{t}(s)+\varepsilon(\gamma(s)) \cdot \tau(s) \cdot \boldsymbol{b}(s) \\
\boldsymbol{b}^{\prime}(s)=\tau(s) \cdot \boldsymbol{n}(s)
\end{array}\right.
$$

where $\tau(s)$ is the torsion of the curve $\gamma$ at $s$ (cf., [7]). This is the fundamental formula for the study of nonlightlike curves in $\mathbf{R}_{1}^{3}$.

We now define

$$
\begin{aligned}
& H_{1}^{2}(r)_{p}=\left\{x=\left(x_{1}, x_{2}, x_{3}\right) \in \mathbf{R}_{1}^{3} \mid\langle x-p, x-p\rangle=-r^{2}\right\}, \\
& S_{1}^{2}(r)_{p}=\left\{x=\left(x_{1}, x_{2}, x_{3}\right) \in \mathbf{R}_{1}^{3} \mid\langle x-p, x-p\rangle=r^{2}\right\},
\end{aligned}
$$

where $p=\left(p_{1}, p_{2}, p_{3}\right)$ and $r \in \mathbf{R}-\{0\}$. We respectively call $H_{1}^{2}(r):=H_{1}^{2}(r)_{p}-\{p\}$, $S_{1}^{2}(r):=S_{1}^{2}(r)_{p}-\{p\}$ a hyperbola, a pseudo sphere with radius $\tau$ at the center $p$. We denote that $H_{1}^{2}=H_{1}^{2}(1)_{0}$ and $S_{1}^{2}=S_{1}^{2}(1)_{0}$.

Let $\gamma: I \rightarrow \mathbf{R}_{1}^{3}$ be a nonlightlike curve with $\varepsilon(\gamma) \delta(\gamma(s))=-1$ and $k(s) \neq 0$. In this case we always have $b(s) \in S_{1}^{2}$. On the other hand, if $\varepsilon(\gamma) \delta(\gamma(s))=1$ and $k(s) \neq 0$, then $\gamma$ is spacelike. In this case, we have $b(s) \in H_{1}^{2}$.

We now define a curve $B N_{\gamma}: I \rightarrow S_{1}^{2}$ for $\varepsilon(\gamma) \delta(\gamma(s))=-1$ (respectively, $B N_{\gamma}: I \rightarrow$ $H_{1}^{2}$ for $\gamma$ with $\left.\varepsilon(\gamma) \delta(\gamma(s))=1\right)$ by

$$
B N_{\gamma}(s)=b(s)
$$


and a ruled surface $E_{\gamma}: I \times \mathbf{R} \rightarrow \mathbf{R}_{1}^{3}$ by

$$
E_{\gamma}(s, u)=\left(\gamma+\frac{\varepsilon(\gamma)}{\delta(\gamma) k} \boldsymbol{n}+u \boldsymbol{b}\right)(s) \text {. }
$$

We call $B N_{\gamma}$ the binormal indicatrix and $E_{\gamma}$ the focal surface of $\gamma$.

In $\S 3$, under the assumption that $k(s) \neq 0, \tau(s) \neq 0$ and $\delta\left(\gamma\left(s_{0}\right)\right)\left(k^{2} \tau^{2}-\varepsilon(\gamma) k^{\prime 2}\right)\left(s_{0}\right)>$ 0 , we show that there exist a unique pseudo sphere which has four-point contact with nonlightlike curve $\gamma$ at $\gamma\left(s_{0}\right)$ which is called the osculating pseudo sphere of $\gamma$ at $s_{0}$ and its center $v=$ $\left(\gamma+(\varepsilon(\gamma) / \delta(\gamma) k) n+\left(k^{\prime} / \varepsilon(\gamma) \delta(\gamma) k^{2} \tau\right) b\right)\left(s_{0}\right)$ is called the center of the osculating pseudo sphere at $s_{0}$. Under the assumption that $k(s) \neq 0, \tau(s) \neq 0,\left\langle\boldsymbol{b}\left(s_{0}\right), \boldsymbol{b}\left(s_{0}\right)\right\rangle=-\delta\left(\gamma\left(s_{0}\right)\right)$ and $\delta\left(\gamma\left(s_{0}\right)\right)\left(k^{2} \tau^{2}-\varepsilon(\gamma) k^{\prime 2}\right)\left(s_{0}\right)<0$, we also show that there exists a unique hyperbola which has four-point contact with spacelike curve $\gamma$ at $\gamma\left(s_{0}\right)$, which is called the osculating hyperbola of $\gamma$ at $s_{0}$ and its center $v=\left(\gamma+(1 / \delta(\gamma) k) n+\left(k^{\prime} / \delta(\gamma) k^{2} \tau\right) b\right)\left(s_{0}\right)$ is called the center of the osculating hyperbola at $s_{0}$.

We now consider the following conditions:

(A 1) The number of points $p$ of $\gamma(I)$ where the osculating pseudo sphere at $p$ having five-point contact with the curve $\gamma$ is finite.

(A 2) There is no point $p$ of $\gamma(I)$ where the osculating pseudo sphere at $p$ having greater than or equal to six-point contact with the curve $\gamma$.

(A 3) The number of points $p$ of $\gamma(I)$ where the osculating hyperbola at $p$ having five-point contact with the curve $\gamma$ is finite.

(A 4) There is no point $p$ of $\gamma(I)$ where the osculating hyperbola at $p$ having greater than six-point contact with the curve $\gamma$.

(A 5) The number of points at where $\gamma$ has at least four-point contact with the osculating plane is finite.

(A 6) There are no osculating plane with which $\gamma$ has five-point contact at a point.

REMARK. If the curve $\gamma$ satisfies the condition $\delta(\gamma(s))\left(k^{2} \tau^{2}-\varepsilon(\gamma) k^{2}\right)(s)>0$ (respectively, $<0$ ), then we can show that there eixsts the osculating pseudo sphere (respectively, osculating hyperbola) at each point of $\gamma$ (cf. §3).

Our main results are formulated as follows:

THEOREM A. (1) Let n-Imm $m_{+}\left(I, \mathbf{R}_{1}^{3}\right)$ be the space of proper nonlightlike curves with $k(s) \neq 0$ and $\delta(\gamma(s))\left(k^{2} \tau^{2}-\varepsilon(\gamma) k^{\prime 2}\right)(s)>0$ equipped with Whitney $C^{\infty}$-topology. Then the set of curves which satisfy (A 1) and (A 2) is a residual set in $n$-Imm $m_{+}\left(I, \mathbf{R}_{1}^{3}\right)$.

(2) Let $n$-Imm $\left(I, \mathbf{R}_{1}^{3}\right)$ be the space of proper nonlightlike curves with $k(s) \neq 0$, $\langle\boldsymbol{b}(s), \boldsymbol{b}(s)\rangle=-\delta(\gamma(s))$ and $\delta(\gamma(s))\left(k^{2} \tau^{2}-\varepsilon(\gamma) k^{\prime 2}\right)(s)<0$ equipped with Whitney $C^{\infty}$ topology. Then the set of curves which satisfy (A 3) and (A 4) is a residual set in $n-I m m_{-}\left(I, \mathbf{R}_{1}^{3}\right)$.

(3) Let $n-\operatorname{Imm}\left(I, \mathbf{R}_{1}^{3}\right)$ be the space of proper nonlightlike curves with $k(s) \neq 0$ equipped with Whitney $C^{\infty}$-topology. Then the set of curves which satisfy (A 5) and (A 6) is a residual set in $n-\operatorname{Imm}\left(I, \mathbf{R}_{1}^{3}\right)$. 
In order to state the second main theorem, we define the ordinary cusp $C$ and the swallow tail $S W$ defined by

$C=\left\{\left(x_{1}, x_{2}\right) \mid x_{1}^{2}=x_{2}^{3}\right\}, \quad S W=\left\{\left(x_{1}, x_{2}, x_{3}\right) \mid x_{1}=3 u^{4}+u^{2} v, x_{2}=4 u^{3}+2 u v, x_{3}=v\right\}$.

THEOREM B. (1) Let $\gamma: I \rightarrow \mathbf{R}_{1}^{3}$ be a nonlightlike curve with $k(s) \neq 0$ and $\tau(s) \neq$ 0 . Under the assumption of (A 1) and (A 2) (respectively, (A 3) and (A 4)), if p is a point of the focal surface $E_{\gamma}$ of $\gamma$ at $s_{0}$, then locally at $p$, the focal surface is

a) diffeomorphic to $C \times \mathbf{R}$ at $(\gamma+(\varepsilon(\gamma) / \delta(\gamma) k) \boldsymbol{n}+u \boldsymbol{b})\left(s_{0}\right)$ in $\mathbf{R}_{1}^{3}$ if and only if $k^{\prime}\left(s_{0}\right) \neq$ 0 and $u_{0}=\left(\left(k^{\prime} / \varepsilon(\gamma) \delta(\gamma) k^{2} \tau\right) \boldsymbol{b}\right)\left(s_{0}\right)$. Moreover, if $\delta\left(\gamma\left(s_{0}\right)\right)\left(k^{2} \tau^{2}-\varepsilon(\gamma) k^{\prime 2}\right)\left(s_{0}\right)>0$, then the locus of the center of the osculating pseudo sphere $(\gamma+(\varepsilon(\gamma) / \delta(\gamma) k) n+u b)\left(s_{0}\right)$ is the cuspidal edge. If $\delta\left(\gamma\left(s_{0}\right)\right)\left(k^{2} \tau^{2}-\varepsilon(\gamma) k^{2}\right)\left(s_{0}\right)<0$ and $\left\langle\boldsymbol{b}\left(s_{0}\right), \boldsymbol{b}\left(s_{0}\right)\right\rangle=-\delta\left(\gamma\left(s_{0}\right)\right)$, then the locus of the center of the osculating hyperbola $(\gamma+(1 / \delta(\gamma) k) n+u b)\left(s_{0}\right)$ is the cuspidal edge.

b) diffeomorphic to a SW at $\left(\gamma+(\varepsilon(\gamma) / \delta(\gamma) k) n+\left(k^{\prime} / \varepsilon(\gamma) \delta(\gamma) k^{2} \tau\right) b\right)\left(s_{0}\right)$ if and only if $k^{\prime}\left(s_{0}\right) \neq 0$ and $\left(k k^{\prime} \tau^{\prime}-k k^{\prime \prime} \tau+k^{2} \tau^{3}+2 k^{\prime 2} \tau\right)\left(s_{0}\right)=0$.

(2) Let $\gamma: I \rightarrow \mathbf{R}_{1}^{3}$ be a nonlightlike curve with $k(s) \neq 0$. Under the assumption of (A 3) and (A 4), if $p$ is a point of the binormal indicatrix of $\gamma$ at $s_{0}$, then locally at $p$, the binormal indicatrix $B N_{\gamma}$ is

a) diffeomorphic to a line if $\tau\left(s_{0}\right) \neq 0$.

b) diffeomorphic to the ordinary cusp $C$ if $\tau\left(s_{0}\right)=0$ and $\tau^{\prime}\left(s_{0}\right) \neq 0$.

\section{Lorentzian invariant functions on nonlightlike curves.}

In this section we introduce two different families of functions on a nonlightlike curve, which are useful to the study of Lorentzian invariants of nonlightlike curves.

Lorentzian height functions: For a nonlightlike curve $\gamma: I \rightarrow \mathbf{R}_{1}^{3}$, we now define a function $H: I \times S_{1}^{2} \rightarrow \mathbf{R}$ (respectively, $H: I \times H_{1}^{2} \rightarrow \mathbf{R}$ ) by $H(s, v)=\langle\gamma, v\rangle$. We call $H$ a Lorentzian height function. We denote that $h(s)=H_{v_{0}}(s)=H\left(s, v_{0}\right)$ for any fixed $v_{0} \in S_{1}^{2}$ (respectively, $v_{0} \in H_{1}^{2}$ ). We have the following proposition.

PROPOSITION 2.1. Let $\gamma: I \rightarrow \mathbf{R}_{1}^{3}$ be a unit speed nonlightlike curve with $k(s) \neq 0$. If $v \in S_{1}^{2}$ (respectively, $v \in H_{1}^{2}$ ), then

(1) $h^{\prime}\left(s_{0}\right)=0$ if and only if there exist $\lambda, \mu \in \mathbf{R}$ such that $v=\lambda \boldsymbol{n}\left(s_{0}\right)+\mu \boldsymbol{b}\left(s_{0}\right)$ and $\delta(\gamma)\left(\lambda^{2}-\varepsilon(\gamma) \mu^{2}\right)=1$ (respectively, $\left.\delta(\gamma)\left(\lambda^{2}-\varepsilon(\gamma) \mu^{2}\right)=-1\right)$.

(2) $h^{\prime}\left(s_{0}\right)=h^{\prime \prime}\left(s_{0}\right)=0$ if and only if $v= \pm b\left(s_{0}\right)$.

(3) $h^{\prime}\left(s_{0}\right)=h^{\prime \prime}\left(s_{0}\right)=h^{(3)}\left(s_{0}\right)=0$ if and only if $v= \pm b\left(s_{0}\right)$ and $\tau\left(s_{0}\right)=0$.

(4) $h^{\prime}\left(s_{0}\right)=h^{\prime \prime}\left(s_{0}\right)=h^{(3)}\left(s_{0}\right)=h^{(4)}\left(s_{0}\right)=0$ if and only if $v= \pm b\left(s_{0}\right)$ and $\tau\left(s_{0}\right)=$ $\tau^{\prime}\left(s_{0}\right)=0$.

Proof. Let $H: I \times S_{1}^{2} \rightarrow \mathbf{R}$ (respectively, $H: I \times H_{1}^{2} \rightarrow \mathbf{R}$ ) be the Lorentzian height function on the nonlightlike curve $\gamma: I \rightarrow \mathbf{R}_{1}^{3}$. Then we have $\frac{\partial H}{\partial s}=\left\langle\gamma^{\prime}(s), v\right\rangle=\langle t(s), v\rangle$ and $v \in S_{1}^{2}$ (respectively, $v \in H_{1}^{2}$ ). It follows that $\frac{\partial H}{\partial s}=0$ if and only if $\langle v, t\rangle=0$. Then 
there exist $\lambda, \mu \in \mathbf{R}$ such that $v=\lambda \boldsymbol{n}+\mu \boldsymbol{b}$ and $\delta(\gamma) \cdot\left(\lambda^{2}-\varepsilon(\gamma) \mu^{2}\right)=1$ (respectively, $\left.\delta(\gamma)\left(\lambda^{2}-\varepsilon(\gamma) \mu^{2}\right)=-1\right)$. Where $\langle\boldsymbol{b}, \boldsymbol{b}\rangle=-\varepsilon(\gamma) \delta(\gamma)$ by $\langle\boldsymbol{t}, \boldsymbol{t}\rangle=\varepsilon(\gamma)$ and $\langle\boldsymbol{n}, \boldsymbol{n}\rangle=\delta(\gamma)$.

On the other hand, we have $\frac{\partial^{2} H}{\partial s^{2}}$. It follows that $\frac{\partial H}{\partial s}=\frac{\partial^{2} H}{\partial s^{2}}=0$ if and only if $v=$ $\lambda \boldsymbol{n}+\mu \boldsymbol{b},\langle k \cdot \boldsymbol{n}, v\rangle=0$ and $\delta(\gamma) \cdot\left(\lambda^{2}-\varepsilon(\gamma) \mu^{2}\right)=1$ (respectively, $\left.\delta(\gamma)\left(\lambda^{2}-\varepsilon(\gamma) \mu^{2}\right)=-1\right)$. This is equivalent to the condition that $v= \pm b$.

Since $\frac{\partial^{3} H}{\partial s^{3}}=\left\langle k^{\prime} \boldsymbol{n}+k \boldsymbol{n}^{\prime}, v\right\rangle, \frac{\partial H}{\partial s}=\frac{\partial^{2} H}{\partial s^{2}}=\frac{\partial^{3} H}{\partial s^{3}}=0$ if and only if $v= \pm \boldsymbol{b}, \tau=0$.

Moreover, we have $\frac{\partial^{4} H}{\partial s^{4}}=\left\langle t^{(3)}, v\right\rangle$. Then $\frac{\partial H}{\partial s}=\frac{\partial^{2} H}{\partial s^{2}}=\frac{\partial^{3} H}{\partial s^{3}}=\frac{\partial^{4} H}{\partial s^{4}}=0$ if and only if $v= \pm \boldsymbol{b}, \tau=\tau^{\prime}=0$.

Lorentzian distance-squared functions: For a nonlightlike curve $\gamma: I \rightarrow \mathbf{R}_{1}^{3}$, we now define a function

$$
G: I \times \mathbf{R}_{1}^{3} \rightarrow \mathbf{R}
$$

by

$$
G(s, v)=\langle\gamma(s)-v, \gamma(s)-v\rangle .
$$

We call $G$ a Lorentzian distance-squared function. We denote that $g(s)=G_{v_{0}}(s)=G\left(s, v_{0}\right)$ for any fixed $v_{0} \in \mathbf{R}_{1}^{3}$. We also have the following proposition.

Proposition 2.2. Let $\gamma: I \rightarrow \mathbf{R}_{1}^{3}$ be a unit speed nonlightlike curve with $k(s) \neq 0$ and $\tau(s) \neq 0$. Then

(1) $g^{\prime}\left(s_{0}\right)=0$ if and only if there exist $\lambda, \mu \in \mathbf{R}$ such that $\gamma\left(s_{0}\right)-v=\lambda \boldsymbol{n}\left(s_{0}\right)+\mu \boldsymbol{b}\left(s_{0}\right)$.

(2) $g^{\prime}\left(s_{0}\right)=g^{\prime \prime}\left(s_{0}\right)=0$ if and only if $v=(\gamma+(\varepsilon(\gamma) / \delta(\gamma) k) n-\mu b)\left(s_{0}\right)$ for some $\mu \in \mathbf{R}$.

(3) $g^{\prime}\left(s_{0}\right)=g^{\prime \prime}\left(s_{0}\right)=g^{(3)}\left(s_{0}\right)=0$ if and only if $v=(\gamma+(\varepsilon(\gamma) / \delta(\gamma) k) n+$ $\left.\left(k^{\prime} / \varepsilon(\gamma) \delta(\gamma) k^{2} \tau\right) b\right)\left(s_{0}\right)$.

(4) $g^{\prime}\left(s_{0}\right)=g^{\prime \prime}\left(s_{0}\right)=g^{(3)}\left(s_{0}\right)=g^{(4)}\left(s_{0}\right)=0$ if and only if $\left(k k^{\prime} \tau^{\prime}-k k^{\prime \prime} \tau-\varepsilon(\gamma) k^{2} \tau^{3}+\right.$ $\left.2 k^{\prime 2} \tau\right)\left(s_{0}\right)=0$ and $v=\left(\gamma+(\varepsilon(\gamma) / \delta(\gamma) k) n+\left(k^{\prime} / \varepsilon(\gamma) \delta(\gamma) k^{2} \tau\right) b\right)\left(s_{0}\right)$.

(5) $g^{\prime}\left(s_{0}\right)=g^{\prime \prime}\left(s_{0}\right)=g^{(3)}\left(s_{0}\right)=g^{(4)}\left(s_{0}\right)=g^{(5)}\left(s_{0}\right)=0$ if and only if $\left(k k^{\prime} \tau^{\prime}-k k^{\prime \prime} \tau-\right.$ $\left.\varepsilon(\gamma) k^{2} \tau^{3}+2 k^{\prime 2} \tau\right)\left(s_{0}\right)=\left(2 k k^{\prime} \tau^{3}-\varepsilon(\gamma) k k^{\prime} \tau^{\prime \prime}+\varepsilon(\gamma) k k^{(3)} \tau+3 k^{2} \tau^{2} \tau^{\prime}-3 \varepsilon(\gamma) k^{\prime} k^{\prime \prime} \tau-\right.$ $\left.3 \varepsilon(\gamma) k^{\prime 2} \tau^{\prime}\right)\left(s_{0}\right)=0$ and $v=\left(\gamma+(\varepsilon(\gamma) / \delta(\gamma) k) \boldsymbol{n}+\left(k^{\prime} / \varepsilon(\gamma) \delta(\gamma) k^{2} \tau\right) \boldsymbol{b}\right)\left(s_{0}\right)$.

Proof. Let $G: I \times \mathbf{R}_{1}^{3} \rightarrow \mathbf{R}$ be a Lorentzian distance-squared function on the nonlightlike curve $\gamma: I \rightarrow \mathbf{R}_{1}^{3}$. Then we have $\frac{\partial G}{\partial s}=2\left\langle\gamma^{\prime}, \gamma-v\right\rangle$. It follows that $\frac{\partial G}{\partial s}=0$ if and only if $\gamma-v=\lambda \cdot \boldsymbol{n}+\mu \cdot \boldsymbol{b}$.

On the other hand, since $\frac{\partial^{2} G}{\partial s^{2}}=2\left\langle\boldsymbol{t}^{\prime}, \gamma-v\right\rangle+2\langle\boldsymbol{t}, \boldsymbol{t}\rangle=2\{\langle k \cdot \boldsymbol{n}, \gamma-v\rangle+\varepsilon(\gamma)\}, \frac{\partial G}{\partial s}=$ $\frac{\partial^{2} G}{\partial s^{2}}=0$ if and only if $\gamma-v=\lambda \boldsymbol{n}+\mu \boldsymbol{b}$ and $\langle k \cdot \boldsymbol{n}, \lambda \boldsymbol{n}+\mu \boldsymbol{b}\rangle=-\varepsilon(\gamma)$. The last equality is equivalent to the relation that $k \lambda \delta(\gamma)=-\varepsilon(\gamma)$. This means that $v=\gamma+(\varepsilon(\gamma) / \delta(\gamma) k) n-\mu \boldsymbol{b}$ by $k \neq 0$.

Since $\frac{\partial^{3} G}{\partial s^{3}}=2\left\langle(k \cdot n)^{\prime}, \gamma-v\right\rangle, \frac{\partial G}{\partial s}=\frac{\partial^{2} G}{\partial s^{2}}=\frac{\partial^{3} G}{\partial s^{3}}=0$ if and only if $k \neq 0$ and $\left\langle k^{\prime} \cdot \boldsymbol{n}+k \cdot \boldsymbol{n}^{\prime},-(\varepsilon(\gamma) / \delta(\gamma) k) \boldsymbol{n}+\mu \boldsymbol{b}\right\rangle=0$, which is equivalent to the condition that $k \neq 0$ and $k^{\prime}+\varepsilon(\gamma) \delta(\gamma) k^{2} \tau \mu=0$. This means that $v=\gamma+(\varepsilon(\gamma) / \delta(\gamma) k) n+\left(k^{\prime} / \varepsilon(\gamma) \delta(\gamma) k^{2} \tau\right) \boldsymbol{b}$ by $k$ and $\tau$ are not zero. 
Moreover, we have $\frac{\partial^{4} G}{\partial s^{4}}=2\left\langle k^{\prime \prime} \boldsymbol{n}+2 k^{\prime} \boldsymbol{n}^{\prime}+k \boldsymbol{n}^{\prime \prime}, \gamma-v\right\rangle+2\left\langle k^{\prime} \boldsymbol{n}+k \boldsymbol{n}^{\prime}, \boldsymbol{t}\right\rangle$. Then $\frac{\partial^{4} G}{\partial s^{4}}=0$ if and only if $\left\langle\left(k^{\prime \prime}-\varepsilon(\gamma) \delta(\gamma) k^{3}+\varepsilon(\gamma) k \tau^{2}\right) n-3 \varepsilon(\gamma) \delta(\gamma) k k^{\prime} t+\left(2 \varepsilon(\gamma) k^{\prime} \tau+\right.\right.$ $\left.\left.\varepsilon(\gamma) k \tau^{\prime}\right) \boldsymbol{b}, \gamma-v\right\rangle=\delta(\gamma) k^{2}$. Therefore $\frac{\partial G}{\partial s}=\frac{\partial^{2} G}{\partial s^{2}}=\frac{\partial^{3} G}{\partial s^{3}}=\frac{\partial^{4} G}{\partial s^{4}}=0$ if and only if $\left\langle\left(k^{\prime \prime}-\varepsilon(\gamma) \delta(\gamma) k^{3}+\varepsilon(\gamma) k \tau^{2}\right) n-3 \varepsilon(\gamma) \delta(\gamma) k k^{\prime} \boldsymbol{t}+\left(2 \varepsilon(\gamma) k^{\prime} \tau+\varepsilon(\gamma) k \tau^{\prime}\right) \boldsymbol{b},-(\varepsilon(\gamma) / \delta(\gamma) k) \boldsymbol{n}-\right.$ $\left.\left(k^{\prime} / \varepsilon(\gamma) \delta(\gamma) k^{2} \tau\right) \boldsymbol{b}\right\rangle=\delta(\gamma) k^{2}$ and $v=\gamma+(\varepsilon(\gamma) / \delta(\gamma) k) n+\left(k^{\prime} / \varepsilon(\gamma) \delta(\gamma) k^{2} \tau\right) b$, which is equivalent to the condition that $k k^{\prime} \tau^{\prime}-k k^{\prime \prime} \tau-\varepsilon(\gamma) k^{2} \tau^{3}+2 k^{\prime 2} \tau=0$ and $v=\gamma+$ $(\varepsilon(\gamma) / \delta(\gamma) k) \boldsymbol{n}+\left(k^{\prime} / \varepsilon(\gamma) \delta(\gamma) k^{2} \tau\right) \boldsymbol{b}$.

Finally, we have $\frac{\partial^{5} G}{\partial s^{5}}=0$ if and only if $\left\langle\left\{\left(k^{\prime \prime}-\varepsilon(\gamma) \delta(\gamma) k^{3}+\varepsilon(\gamma) k \tau^{2}\right) n-\right.\right.$ $\left.\left.3 \varepsilon(\gamma) \delta(\gamma) k k^{\prime} t+\left(2 \varepsilon(\gamma) k^{\prime} \tau+\varepsilon(\gamma) k \tau^{\prime}\right) b\right\}^{\prime}, \gamma-v\right\rangle=5 \delta(\gamma) k k^{\prime}$. This is equivalent to the condition that $\left\langle\left(k^{(3)}-3 \varepsilon(\gamma) \delta(\gamma) k^{2} k^{\prime}+\varepsilon(\gamma) k^{\prime} \tau^{2}+3 \varepsilon(\gamma) k \tau \tau^{\prime}-3 \varepsilon(\gamma) \delta(\gamma) k^{2} k^{\prime}+2 \varepsilon(\gamma) k^{\prime} \tau^{2}\right) n+\right.$ $\left(k^{4}-\delta(\gamma) k^{2} \tau^{2}-3 \varepsilon(\gamma) \delta(\gamma) k^{2}-4 \varepsilon(\gamma) \delta(\gamma) k k^{\prime \prime}\right) t+\left(3 \varepsilon(\gamma) k^{\prime \prime} \tau+2 \varepsilon(\gamma) k^{\prime} \tau^{\prime}+\varepsilon(\gamma) k^{\prime} \tau^{\prime}+\right.$ $\left.\left.\varepsilon(\gamma) k \tau^{\prime \prime}-\delta(\gamma) k^{3} \tau+k \tau^{3}\right) b, \gamma-v\right\rangle=5 \delta(\gamma) k k^{\prime}$. So $\frac{\partial G}{\partial s}=\frac{\partial^{2} G}{\partial s^{2}}=\frac{\partial^{3} G}{\partial s^{3}}=\frac{\partial^{4} G}{\partial s^{4}}=\frac{\partial^{5} G}{\partial s^{5}}=0$ if and only if $k k^{\prime} \tau^{\prime}-k k^{\prime \prime} \tau-\varepsilon(\gamma) k^{2} \tau^{3}+2 k^{\prime 2} \tau=2 k k^{\prime} \tau^{3}-\varepsilon(\gamma) k k^{\prime} \tau^{\prime \prime}+\varepsilon(\gamma) k k^{(3)} \tau+3 k^{2} \tau^{2} \tau^{\prime}-$ $3 \varepsilon(\gamma) k^{\prime} k^{\prime \prime} \tau-3 \varepsilon(\gamma) k^{\prime 2} \tau^{\prime}=0$ and $v=\gamma+(\varepsilon(\gamma) / \delta(\gamma) k) n+\left(k^{\prime} / \varepsilon(\gamma) \delta(\gamma) k^{2} \tau\right) b$.

\section{Lorentzian invariants of nonlightlike curves.}

In this section we study the geometric properties of the binormal indicatrix and focal developables of the nonlightlike curves. By the propositions in the last section, we can recognize the function $\left(k k^{\prime} \tau^{\prime}-k k^{\prime \prime} \tau-\varepsilon(\gamma) k^{2} \tau^{3}+2 k^{\prime 2} \tau\right)(s)$ has special meanings. Firstly we have the following proposition.

Proposition 3.1. Let $\gamma: I \rightarrow \mathbf{R}_{1}^{3}$ be a nonlightlike curve with $k(s) \neq 0, k^{\prime}(s) \neq 0$ and $\tau(s) \neq 0$. Then $\left(k k^{\prime} \tau^{\prime}-k k^{\prime \prime} \tau-\varepsilon(\gamma) k^{2} \tau^{3}+2 k^{\prime 2} \tau\right)(s) \equiv 0$ if and only if $p=\gamma(s)+$ $(\varepsilon(\gamma(s)) / \delta(\gamma(s)) k(s)) n(s)+\left(k^{\prime}(s) / \varepsilon(\gamma(s)) \delta(\gamma(s)) k^{2}(s) \tau(s)\right) b(s)$ are constant vectors.

Proof. We put

$$
P(s)=\gamma(s)+\frac{\varepsilon(\gamma(s))}{\delta(\gamma(s)) k(s)} n(s)+\frac{k^{\prime}(s)}{\varepsilon(\gamma(s)) \delta(\gamma(s)) k^{2}(s) \tau(s)} b(s)
$$

then we have

$$
P^{\prime}(s)=-\frac{\left(k k^{\prime} \tau^{\prime}-k k^{\prime \prime} \tau-\varepsilon(\gamma) k^{2} \tau^{3}+2 k^{\prime 2} \tau\right)(s)}{\varepsilon(\gamma(s)) \delta(\gamma(s)) k^{3}(s) \tau^{2}(s)} b(s)
$$

It follows that $P^{\prime}(s) \equiv 0$ if and only if $\left(k k^{\prime} \tau^{\prime}-k k^{\prime \prime} \tau-\varepsilon(\gamma) k^{2} \tau^{3}+2 k^{2} \tau\right)(s) \equiv 0$.

Let $\gamma: I \rightarrow \mathbf{R}_{1}^{3}$ be a nonlightlike curve with $k(s) \neq 0, \tau(s) \neq 0$ and $\delta(\gamma(s))\left(k^{2} \tau^{2}-\right.$ $\left.k^{\prime 2}\right)(s) \neq 0$ (the last condition is always satisfied for timelike curves), and $G: I \times \mathbf{R}_{1}^{3} \rightarrow$ $\mathbf{R}$ a Lorentzian distance-squared function on the curve $\gamma$. Under the conditions $g^{\prime}\left(s_{0}\right)=$ $g^{\prime \prime}\left(s_{0}\right)=g^{(3)}\left(s_{0}\right)=0$ at $s=s_{0}$, we can show that $\left\langle\gamma\left(s_{0}\right)-v, \gamma\left(s_{0}\right)-v\right\rangle=\left(\delta(\gamma)\left(k^{2} \tau^{2}-\right.\right.$ $\left.\left.\varepsilon(\gamma) k^{\prime 2}\right) / k^{4} \tau^{2}\right)\left(s_{0}\right)>0$ for $\delta\left(\gamma\left(s_{0}\right)\right)\left(k^{2} \tau^{2}-\varepsilon(\gamma) k^{\prime 2}\right)\left(s_{0}\right)>0$ and $\left\langle\gamma\left(s_{0}\right)-v, \gamma\left(s_{0}\right)-v\right\rangle<0$ for $\delta\left(\gamma\left(s_{0}\right)\right)\left(k^{2} \tau^{2}-k^{\prime 2}\right)\left(s_{0}\right)<0$ by Proposition 2.2. (For the case $\delta\left(\gamma\left(s_{0}\right)\right)\left(k^{2} \tau^{2}-k^{\prime 2}\right)\left(s_{0}\right)=$ 0 on spacelike curves, see [6].)

So we have the following corollaries. 
COROLlARY 3.2. Let $\gamma: I \rightarrow \mathbf{R}_{1}^{3}$ be a nonlightlike curve with $k(s) \neq 0, k^{\prime}(s) \neq 0$, $\tau(s) \neq 0$ and $\delta(\gamma(s))\left(k^{2} \tau^{2}-\varepsilon(\gamma) k^{\prime 2}\right)(s)>0$. Then singularities of focal surface are the locus of centers of osculating pseudo spheres of $\gamma$ which is given by $p(s)=\gamma(s)+$ $(\varepsilon(\gamma(s)) / \delta(\gamma(s)) k(s)) \boldsymbol{n}(s)+\left(k^{\prime}(s) / \varepsilon(\gamma(s)) \delta(\gamma(s)) k^{2}(s) \tau(s)\right) \boldsymbol{b}(s)$. Moreover, if $\left(k k^{\prime} \tau^{\prime}-\right.$ $\left.k k^{\prime \prime} \tau-\varepsilon(\gamma) k^{2} \tau^{3}+2 k^{\prime 2} \tau\right)(s) \equiv 0$, then $p(s)$ is a constant point and $\gamma$ is a curve on the pseudo sphere whose center is $p(s)$ and radius is $r=\sqrt{\delta(\gamma)\left(k^{2} \tau^{2}-\varepsilon(\gamma) k^{\prime 2}\right) / k^{4} \tau^{2}}(s)$.

COROLLARY 3.3. Let $\gamma: I \rightarrow \mathbf{R}_{1}^{3}$ be a spacelike curve with $k(s) \neq 0, k^{\prime}(s) \neq$ $0, \tau(s) \neq 0$ and $\delta(\gamma(s))\left(k^{2} \tau^{2}-k^{2}\right)(s)<0$. Then singularities of focal surface are the locus of centers of osculating pseudo hyperbolas of $\gamma$ which is given by $p(s)=\gamma(s)+$ $(1 / \delta(\gamma(s)) k(s)) n(s)+\left(k^{\prime}(s) / \delta(\gamma(s)) k^{2}(s) \tau(s)\right) b(s)$. Moreover, if $\left(k k^{\prime} \tau^{\prime}-k k^{\prime \prime} \tau-k^{2} \tau^{3}+\right.$ $\left.2 k^{\prime 2} \tau\right) \equiv 0$, then $p(s)$ is a constant point and $\gamma$ is a curve on the osculating hyperbola whose center is $p(s)$ and radius is $r=\sqrt{-\delta(\gamma)\left(k^{2} \tau^{2}-k^{\prime 2}\right) / k^{4} \tau^{2}}(s)$.

Let $F: \mathbf{R}_{1}^{3} \rightarrow \mathbf{R}$ be a submersion and $\gamma: I \rightarrow \mathbf{R}_{1}^{3}$ be a nonlightlike curve. We say that $\gamma$ and $F^{-1}(0)$ have $k$-point contact for $t=t_{0}$ if the function $g(t)=F \circ \gamma(t)$ satisfies $g\left(t_{0}\right)=g^{\prime}\left(t_{0}\right)=\cdots=g^{(k-1)}\left(t_{0}\right)=0, g^{(k)}\left(t_{0}\right) \neq 0$. By Propositions 2.1, 2.2 and 3.1, we have the following proposition.

PROPOSITION 3.4. (1) Let $\gamma: I \rightarrow \mathbf{R}_{1}^{3}$ be a nonlightlike curve with $k(s) \neq 0$. Then the binormal $b$ and the osculating plane of $\gamma$ have 3-point contact for $s=s_{0}$ if and only if $\tau\left(s_{0}\right) \equiv 0$ and $\tau^{\prime}\left(s_{0}\right) \neq 0$.

(2) Let $\gamma: I \rightarrow \mathbf{R}_{1}^{3}$ be a nonlightlike curve with $k(s) \neq 0, k^{\prime}(s) \neq 0$ and $\tau(s) \neq$ 0 . Then $\gamma$ and the osculating pseudo sphere have 4-point contact for $s=s_{0}$ if and only if $\delta\left(\gamma\left(s_{0}\right)\right)\left(k^{2} \tau^{2}-\varepsilon(\gamma) k^{2}\right)\left(s_{0}\right)>0,\left(k k^{\prime} \tau^{\prime}-k k^{\prime \prime} \tau-\varepsilon(\gamma) k^{2} \tau^{3}+2 k^{\prime 2} \tau\right)\left(s_{0}\right)=0$ and $\left(2 k k^{\prime} \tau^{3}-\varepsilon(\gamma) k k^{\prime} \tau^{\prime \prime}+\varepsilon(\gamma) k k^{(3)} \tau+3 k^{2} \tau^{2} \tau^{\prime}-3 \varepsilon(\gamma) k^{\prime} k^{\prime \prime} \tau-3 \varepsilon(\gamma) k^{\prime 2} \tau^{\prime}\right)\left(s_{0}\right) \neq 0$.

(3) Let $\gamma: I \rightarrow \mathbf{R}_{1}^{3}$ be a spacelike curve with $k(s) \neq 0, k^{\prime}(s) \neq 0$ and $\tau(s) \neq 0$. Then $\gamma$ and the osculating hyperbola have 4-point contact for $s=s_{0}$ if and only if $\delta\left(\gamma\left(s_{0}\right)\right)\left(k^{2} \tau^{2}-\right.$ $\left.\varepsilon(\gamma) k^{\prime 2}\right)\left(s_{0}\right)<0,\left(k k^{\prime} \tau^{\prime}-k k^{\prime \prime} \tau-k^{2} \tau^{3}+2 k^{\prime 2} \tau\right)\left(s_{0}\right)=0$ and $\left(2 k k^{\prime} \tau^{3}-k k^{\prime} \tau^{\prime \prime}+k k^{(3)} \tau+\right.$ $\left.3 k^{2} \tau^{2} \tau^{\prime}-3 k^{\prime} k^{\prime \prime} \tau-3 k^{\prime 2} \tau^{\prime}\right)\left(s_{0}\right) \neq 0$.

\section{Unfoldings of functions by one-variable.}

In this section we use some general results on the singularity theory for families of function germs. Detailed descriptions are found in the book [4]. Let $F:\left(\mathbf{R} \times \mathbf{R}^{r},\left(s_{0}, x_{0}\right)\right) \rightarrow \mathbf{R}$ be a function germ. We call $F$ an $r$-parameter unfolding of $f$, where $f(s)=F_{x_{0}}\left(s, x_{0}\right)$. We say that $f$ has $A_{k}$-singularity at $s_{0}$ if $f^{(p)}\left(s_{0}\right)=0$ for all $1 \leqq p \leqq k$, and $f^{(k+1)}\left(s_{0}\right) \neq 0$. We also say that $f$ has $A_{\geq k}$-singularity at $s_{0}$ if $f^{(p)}\left(s_{0}\right)=0$ for all $1 \leqq p \leqq k$. Let $F$ be an unfolding of $f$ and $f(s)$ has $A_{k}$-singularity $(k \geqq 1)$ at $s_{0}$. We denote the $(k-1)$-jet of the partial derivative $\frac{\partial F}{\partial x_{i}}$ at $s_{0}$ by $j^{(k-1)}\left(\frac{\partial F}{\partial x_{i}}\left(s, x_{0}\right)\right)\left(s_{0}\right)=\sum_{j=1}^{k-1} \alpha_{j i} s^{j}$ for $i=1, \cdots, r$. Then $F$ is called a $(p)$ versal unfolding if the $(k-1) \times r$ matrix of coefficients $\left(\alpha_{j i}\right)$ has rank $k-1$ $(k-1 \leqq r)$. There is an important set concerning the unfoldings relative to the above notions. 
The bifurcation set of $F$ is the set

$$
\mathfrak{B}_{F}=\left\{x \in \mathbf{R}^{r} \mid \text { there exists } s \text { with } \frac{\partial F}{\partial s}=\frac{\partial^{2} F}{\partial s^{2}}=0 \text { at }(s, x)\right\} .
$$

Then we have the following well-known theorem (cf. [4]).

THEOREM 4.1. Let $F:\left(\mathbf{R} \times \mathbf{R}^{r},\left(s_{0}, x_{0}\right)\right) \rightarrow \mathbf{R}$ be an $r$-parameter unfolding of $f(s)$ which has the $A_{k}$-singularity at $s_{0}$.

Suppose that $F$ is an $(p)$ versal unfolding.

(a) If $k=2$, then $\mathfrak{B}_{F}$ is diffeomorphic to $\{0\} \times \mathbf{R}^{r-1}$.

(b) If $k=3$, then $\mathfrak{B}_{F}$ is diffeomorphic to $C \times \mathbf{R}^{r-2}$.

(c) If $k=4$, then $\mathfrak{B}_{F}$ is diffeomorphic to $S W \times \mathbf{R}^{r-3}$.

Where, $C=\left\{\left(x_{1}, x_{2}\right) \mid x_{1}{ }^{2}=x_{2}{ }^{3}\right\}$ and $S W=\left\{\left(x_{1}, x_{2}, x_{3}\right) \mid x_{1}=3 u^{4}+u^{2} v, x_{2}=4 u^{3}+\right.$ $\left.2 u v, x_{3}=v\right\}$.

For the proof of Theorem B we have the following fundamental theorem.

THEOREM 4.2. (1) Let $\gamma: I \rightarrow \mathbf{R}_{1}^{3}$ be a unit speed nonlightlike curve with $k(s) \neq 0$ and $H: I \times S_{1}^{2} \rightarrow \mathbf{R}$ be the height function on $\gamma$.

If $h(s)=H_{v_{0}}(s)$ has the $A_{k}$-singularity $(k=2,3)$ at $s_{0}$, then $H$ is the (p)versal unfolding of $h$.

(2) Let $\gamma: I \rightarrow \mathbf{R}_{1}^{3}$ be a unit speed spacelike curve with $k(s) \neq 0$ and $H: I \times H_{1}^{2} \rightarrow \mathbf{R}$ be the height function on $\gamma$.

If $h(s)=H_{v_{0}}(s)$ has the $A_{k}$-singularity $(k=2,3)$ at $s_{0}$, then $H$ is the $(p)$ versal unfolding of $h$.

(3) Let $\gamma: I \rightarrow \mathbf{R}_{1}^{3}$ be a unit speed nonlightlike curve with $k(s) \neq 0$ and $G: I \times \mathbf{R}_{1}^{3} \rightarrow$ $\mathbf{R}$ be the distance-squared function. We consider the point $\left(s_{0}, v_{0}\right) \in I \times \mathbf{R}_{1}^{3}$.

If $g(s)=G_{v_{0}}(s)$ has the $A_{k}$-singularity $(k=2,3,4)$ at $s_{0}$, then $G$ is the $(p)$ versal unfolding of $g:=G_{v_{0}}$.

PROOF. (1) Let $\gamma(s)=\left(x_{1}(s), x_{2}(s), x_{3}(s)\right)$ be a nonlightlike curve and $v=$ $\left(v_{1}, v_{2}, v_{3}\right) \in S_{1}^{2}$. By definition, in this case we have

$$
H(s, v)=-v_{1} x_{1}(s)+v_{2} x_{2}(s)+v_{3} x_{3}(s)=-v_{1} x_{1}(s) \pm \sqrt{1+v_{1}^{2}-v_{3}^{2}} x_{2}(s)+v_{3} x_{3}(s) .
$$

So that $\frac{\partial H}{\partial v_{1}}=-x_{1}(s) \pm v_{1} x_{2}(s) / \sqrt{1+v_{1}^{2}-v_{3}^{2}}=-x_{1}(s)+\left(v_{1} / v_{2}\right) x_{2}(s)$, (since $v$ is spacelike, $v_{2}$ and $v_{3}$ are not both zero, so we let $v_{2} \neq 0$ ) so the 2-jet at $s_{0}$ is $-\left(s x_{1}^{\prime}(s)+(1 / 2) s^{2} x_{1}^{\prime \prime}(s)\right)+\left(v_{1} / v_{2}\right)\left(s x_{1}^{\prime}(s)+(1 / 2) s^{2} x_{1}^{\prime \prime}(s)\right)$. Wealsohave $\frac{\partial H}{\partial v_{3}}=\mp v_{3} x_{2}(s) /$ $\sqrt{1+v_{1}^{2}-v_{3}^{2}}+x_{3}=-\left(v_{3} / v_{2}\right) x_{2}+x_{3}$, so the 2-jet at $s_{0}$ is $\left(s x_{3}^{\prime}\left(s_{0}\right)+(1 / 2) s^{2} x_{3}^{\prime \prime}\left(s_{0}\right)\right)-$ $\left(v_{3} / v_{2}\right)\left(s x_{2}^{\prime}\left(s_{0}\right)+(1 / 2) s^{2} x_{2}^{\prime \prime}\left(s_{0}\right)\right)$.

(i) By Proposition $2.1, h$ has the $A_{2}$-singularity at $s_{0}$ if and only if $v\left(s_{0}\right)= \pm b\left(s_{0}\right)$ and $\tau\left(s_{0}\right) \neq 0$. When $h$ has $A_{2}$ at $s_{0}$, we require the $1 \times 2$ matrix $\left(-x_{1}^{\prime}(s)+\left(v_{1} / v_{2}\right) x_{2}^{\prime}(s)\right.$, $\left.-\left(v_{3} / v_{2}\right) x_{2}^{\prime}+x_{3}^{\prime}\right)$ to have rank 1 , which follows from the proof of the case (ii). 
(ii) By Proposition 2.1, $h$ has $A_{3}$ at $s_{0}$ if and only if $v= \pm \boldsymbol{b}\left(s_{0}\right), \tau\left(s_{0}\right)=0$ and $\tau^{\prime}\left(s_{0}\right) \neq 0$. When $h$ has $A_{3}$ at $s$, we require the $2 \times 2$ matrix

$$
\left(\begin{array}{cc}
-x_{1}^{\prime}(s)+\frac{v_{1}}{v_{2}} x_{2}^{\prime}(s) & -\frac{v_{3}}{v_{2}} x_{2}^{\prime}+x_{3} \\
-\frac{1}{2} x_{1}^{\prime \prime}(s)+\frac{v_{1}}{2 v_{2}} x_{2}^{\prime \prime}(s) & -\frac{v_{3}}{2 v_{2}} x_{2}^{\prime \prime}+\frac{1}{2} x_{3}^{\prime \prime}
\end{array}\right)
$$

to be nonsingular, which it always does. In fact, by $v= \pm \boldsymbol{b}\left(s_{0}\right), v\left(s_{0}\right)= \pm \boldsymbol{t}\left(s_{0}\right) \wedge \boldsymbol{n}\left(s_{0}\right)=$ $\pm\left(1 / k\left(s_{0}\right)\right)\left(\gamma^{\prime}\left(s_{0}\right) \wedge \gamma^{\prime \prime}\left(s_{0}\right)\right)=\left(v_{1}, v_{2}, v_{3}\right) \in S_{1}^{2}$. So that

$$
\left\{\begin{array}{l}
v_{1}= \pm \frac{1}{k}\left(-x_{2}^{\prime} x_{3}^{\prime \prime}+x_{3}^{\prime} x_{2}^{\prime \prime}\right) \\
v_{2}= \pm \frac{1}{k}\left(x_{3}^{\prime} x_{1}^{\prime \prime}-x_{1}^{\prime} x_{3}^{\prime \prime}\right) \\
v_{3}= \pm \frac{1}{k}\left(x_{1}^{\prime} x_{2}^{\prime \prime}-x_{2}^{\prime} x_{1}^{\prime \prime}\right) .
\end{array}\right.
$$

Hence, the determinant of the above matrix is $(1 / 2)\left\{\left(x_{3}^{\prime} x_{1}^{\prime \prime}-x_{1}^{\prime} x_{3}^{\prime \prime}\right)+\left(v_{3} / v_{2}\right)\left(x_{1}^{\prime} x_{2}^{\prime \prime}-x_{2}^{\prime} x_{1}^{\prime \prime}\right)-\right.$ $\left.\left(v_{1} / v_{2}\right)\left(-x_{2}^{\prime} x_{3}^{\prime \prime}+x_{3}^{\prime} x_{2}^{\prime \prime}\right)\right\}= \pm(k / 2)\left(v_{2}+v_{3}^{2} / v_{2}-v_{1}^{2} / v_{2}\right)= \pm k / 2 v_{2} \neq 0$ by $k\left(s_{0}\right) \neq 0$ and $v_{2} \neq 0$. This means that $H$ is (p)versal when $h(s)$ has the $A_{k}$-singularity $(k=2,3)$ at $s_{0}$.

(2) Let $\gamma(s)=\left(x_{1}(s), x_{2}(s), x_{3}(s)\right)$ be a spacelike curve and $v=\left(v_{1}, v_{2}, v_{3}\right) \in H_{1}^{2}$. By definition, in this case we have

$$
H(s, v)=-v_{1} x_{1}(s)+v_{2} x_{2}(s)+v_{3} x_{3}(s)=\mp \sqrt{1+v_{2}^{2}+v_{3}^{2}} x_{1}(s)+v_{2} x_{2}(s)+v_{3} x_{3}(s) .
$$

So that $\frac{\partial H}{\partial v_{2}}=+x_{2}(s) \mp v_{2} x_{1}(s) / \sqrt{1+v_{2}^{2}+v_{3}^{2}}=-\left(v_{2} / v_{1}\right) x_{1}(s)+x_{2}(s)$, so the 2-jet at $s_{0}$ is $\left(s x_{2}^{\prime}(s)+(1 / 2) s^{2} x_{2}^{\prime \prime}(s)\right)-\left(v_{2} / v_{1}\right)\left(s x_{1}^{\prime}(s)+(1 / 2) s^{2} x_{1}^{\prime \prime}(s)\right)$. We also have $\frac{\partial H}{\partial v_{3}}=\mp v_{3} x_{1}(s) / \sqrt{1+v_{2}^{2}+v_{3}^{2}}+x_{3}=-\left(v_{3} / v_{1}\right) x_{1}+x_{3}$, so the 2-jet at $s_{0}$ is $\left(s x_{3}^{\prime}\left(s_{0}\right)+\right.$ $\left.(1 / 2) s^{2} x_{3}^{\prime \prime}\left(s_{0}\right)\right)-\left(v_{3} / v_{1}\right)\left(s x_{1}^{\prime}\left(s_{0}\right)+(1 / 2) s^{2} x_{1}^{\prime \prime}\left(s_{0}\right)\right)$.

(i) By Proposition 2.1, $h$ has the $A_{2}$-singularity at $s_{0}$ if and only if $v\left(s_{0}\right)= \pm \boldsymbol{b}\left(s_{0}\right)$ and $\tau\left(s_{0}\right) \neq 0$. When $h$ has $A_{2}$ at $s_{0}$, we require the $1 \times 2$ matrix $\left(-\left(v_{2} / v_{1}\right) x_{1}^{\prime}(s)+\right.$ $\left.x_{2}^{\prime}(s),-\left(v_{3} / v_{1}\right) x_{1}^{\prime}+x_{3}^{\prime}\right)$ to have rank 1 , which follows from the proof of the case (ii).

(ii) By Proposition 2.1, $h$ has $A_{3}$ at $s_{0}$ if and only if $v= \pm b\left(s_{0}\right), \tau\left(s_{0}\right)=0$ and $\tau^{\prime}\left(s_{0}\right) \neq 0$. When $h$ has $A_{3}$ at $s$, we require the $2 \times 2$ matrix

$$
\left(\begin{array}{cc}
-\frac{v_{2}}{v_{1}} x_{1}^{\prime}(s)+x_{2}^{\prime}(s) & -\frac{v_{3}}{v_{1}} x_{1}^{\prime}+x_{3}^{\prime} \\
-\frac{v_{2}}{2 v_{1}} x_{1}^{\prime \prime}(s)+\frac{1}{2} x_{2}^{\prime \prime}(s) & -\frac{v_{3}}{2 v_{1}} x_{1}^{\prime \prime}+\frac{1}{2} x_{3}^{\prime \prime}
\end{array}\right)
$$

to be nonsingular, which it always does. By the same reason as the case 1), the determinant of the above matrix is $(1 / 2)\left\{\left(v_{2} / v_{1}\right)\left(x_{3}^{\prime} x_{1}^{\prime \prime}-x_{1}^{\prime} x_{3}^{\prime \prime}\right)+\left(v_{3} / v_{1}\right)\left(x_{1}^{\prime} x_{2}^{\prime \prime}-x_{2}^{\prime} x_{1}^{\prime \prime}\right)-\left(-x_{2}^{\prime} x_{3}^{\prime \prime}+x_{3}^{\prime} x_{2}^{\prime \prime}\right)\right\}=$ $\pm(k / 2)\left(\left(v_{2}^{2} / v_{1}\right)+\left(v_{3}^{2} / v_{1}\right)-v_{1}\right)=\mp k / 2 v_{1} \neq 0$ by $k\left(s_{0}\right) \neq 0$ and $v \in H_{1}^{2}$. This means that $H$ is (p)versal when $h(s)$ has the $A_{k}$-singularity $(k=2,3)$ at $s_{0}$.

(3) In this case we have

$$
G(s, v)=-\left(x_{1}(s)-v_{1}\right)^{2}+\left(x_{2}(s)-v_{2}\right)^{2}+\left(x_{3}(s)-v_{3}\right)^{2},
$$

where $\gamma(s)=\left(x_{1}(s), x_{2}(s), x_{3}(s)\right)$ and $v=\left(v_{1}, v_{2}, v_{3}\right)$. 
Thus we have $\frac{\partial G}{\partial v_{1}}(s)=2\left(x_{1}(s)-v_{1}\right)$, so the 2 -jet at $s_{0}$ is $2\left(s x_{1}^{\prime}\left(s_{0}\right)+(1 / 2) s^{2} x_{1}^{\prime \prime}\left(s_{0}\right)\right)$ and the 3 -jet at $s_{0}$ is $2\left(s x_{1}^{\prime}\left(s_{0}\right)+(1 / 2) s^{2} x_{1}^{\prime \prime}\left(s_{0}\right)+(1 / 3) s^{3} x_{1}{ }^{(3)}\left(s_{0}\right)\right)$. We also have $\frac{\partial G}{\partial v_{i}}(s)=$ $-2\left(x_{i}(s)-v_{i}\right)(i=2,3)$, so the 2-jet at $s_{0}$ is $-2\left(s x_{i}^{\prime}\left(s_{0}\right)+(1 / 2) s^{2} x_{i}^{\prime \prime}\left(s_{0}\right)\right)$ and the 3-jet at $s_{0}$ is $-2\left(s x_{i}^{\prime}\left(s_{0}\right)+(1 / 2) s^{2} x_{i}^{\prime \prime}\left(s_{0}\right)+(1 / 3) s^{3} x_{i}{ }^{(3)}\left(s_{0}\right)\right)$. The condition for (p)versality can be checked as follows:

(i) By Proposition 2.2, $g$ has the $A_{\geq 2}$-singularity at $s_{0}$ if and only if $v=\gamma\left(s_{0}\right)+$ $\left(\varepsilon\left(\gamma\left(s_{0}\right)\right) / \delta\left(\gamma\left(s_{0}\right)\right) k\left(s_{0}\right)\right) \boldsymbol{n}\left(s_{0}\right)-\mu \boldsymbol{b}\left(s_{0}\right)$ and $k\left(s_{0}\right), \tau\left(s_{0}\right) \neq 0$. When $g$ has the $A_{\geq 2}$-singularity at $s_{0}$, we require the $1 \times 3$ matrix $\left(2 x_{1}^{\prime}\left(s_{0}\right),-2 x_{2}^{\prime}\left(s_{0}\right),-2\left(x_{3}^{\prime}\left(s_{0}\right)\right)\right)$ to have rank 1 , which it always does since $\gamma$ is regular.

(ii) It also follows from Proposition 2.2 that $g$ has the $A_{\geq 3}$-singularity at $s_{0}$ if and only if $v=\left(\gamma+(\varepsilon(\gamma) / \delta(\gamma) k) n+\left(k^{\prime} / \varepsilon(\gamma) \delta(\gamma) k^{2} \tau\right) b\right)\left(s_{0}\right)$ and $k\left(s_{0}\right), \tau\left(s_{0}\right) \neq 0$. When $g$ has $A_{2}$ at $s_{0}$, we require the $2 \times 3$ matrix

$$
\left(\begin{array}{ccc}
2 x_{1}^{\prime}\left(s_{0}\right) & -2 x_{2}^{\prime}\left(s_{0}\right) & -2 x_{3}^{\prime}\left(s_{0}\right) \\
x_{1}^{\prime \prime}\left(s_{0}\right) & -x_{2}^{\prime \prime}\left(s_{0}\right) & -x_{3}^{\prime \prime}\left(s_{0}\right)
\end{array}\right)
$$

to have rank 2, which follows from the proof of the case (iii).

(iii) By Proposition 2.2, $g$ has the $A_{\geq 4}$-singularity at $s_{0}$ if and only if $\left(k k^{\prime} \tau^{\prime}-k k^{\prime \prime} \tau-\right.$ $\left.\varepsilon(\gamma) k^{2} \tau^{3}+2 k^{\prime 2} \tau\right)\left(s_{0}\right)=0$ and $v=\left(\gamma+(\varepsilon(\gamma) / \delta(\gamma) k) n+\left(k^{\prime} / \varepsilon(\gamma) \delta(\gamma) k^{2} \tau\right) b\right)\left(s_{0}\right)$. Where $k\left(s_{0}\right), \tau \neq 0$. When $g$ has $A_{\geq 4}$ at $s_{0}$, we require the $3 \times 3$ matrix

$$
\left(\begin{array}{ccc}
2 x_{1}^{\prime}\left(s_{0}\right) & -2 x_{2}^{\prime}\left(s_{0}\right) & -2 x_{3}^{\prime}\left(s_{0}\right) \\
x_{1}^{\prime \prime}\left(s_{0}\right) & -x_{2}^{\prime \prime}\left(s_{0}\right) & -x_{3}^{\prime \prime}\left(s_{0}\right) \\
\frac{2}{3} x_{1}^{(3)}\left(s_{0}\right) & -\frac{2}{3} x_{2}^{(3)}\left(s_{0}\right) & -\frac{2}{3} x_{3}^{(3)}\left(s_{0}\right)
\end{array}\right)
$$

to be nonsingular, which it always does since the determinant of the above matrix is $(4 / 3) \varepsilon\left(\gamma\left(s_{0}\right)\right) k^{2}\left(s_{0}\right) \tau\left(s_{0}\right) \neq 0$ by $k\left(s_{0}\right) \neq 0$ and $\tau\left(s_{0}\right) \neq 0$. This completes the proof.

For the proof of Theorem B, we consider the bifurcation set of $H$ and $G$. By Propositions 2.1 and 2.2, the bifurcation set of $H$ is

$$
\mathfrak{B}_{H}=\left\{v \in \mathbf{R}_{1}^{3} \mid v=\boldsymbol{b}(s)\right\},
$$

and the bifurcation set of $G$ is

$$
\mathfrak{B}_{G}=\left\{v \in \mathbf{R}_{1}^{3} \mid v=\gamma(s)+(\varepsilon(\gamma(s)) / \delta(\gamma(s)) k(s)) \boldsymbol{n}(s)+u \boldsymbol{b}(s)\right.
$$

with the real number $u$ is not zero\}.

The assertions of Theorem B follow from Propositions 2.1, 2.2 and Theorems 4.1, 4.2.

\section{Generic properties of nonlightlike curves.}

We now consider the notion of Lorentzian Monge-Taylor maps for timelike curves analogous to the ordinary notion of Monge-Taylor maps for space curves in Euclidean space (cf. [4]). (For spacelike curves, see [6].) Let $\gamma: I \rightarrow \mathbf{R}_{1}^{3}$ be a (regular) timelike curve, with $I$ an 
open connected subset of the unit circle $S^{1}$, increasing $t$ corresponding the anticlockwise orientation of $S^{1}$. We now choose a smooth family of unit vectors $\boldsymbol{n}(t)$, with $\boldsymbol{n}(t)$ pseudonormal to $\gamma$ at $t$, so $\|\boldsymbol{n}(t)\|=1$ and $\langle\boldsymbol{n}(t), t(t)\rangle=0$ for all $t \in I$. Such $n(t)$ can be obtained as follows: consider the smooth map $t: I \rightarrow S_{1}^{2}$ which takes $t$ to the unit tangent vector $t(t)$. If $V$ is any vector in $S_{1}^{2}$ we can obtain the vector field $\boldsymbol{n}(t)$ by pseudo-orthogonally projecting $V$ onto each of the normal planes and normalizing. Thus $\boldsymbol{n}(t)=(V+\langle V, \boldsymbol{t}(t)\rangle \boldsymbol{t}(t)) /\|V+\langle V, \boldsymbol{t}(t)\rangle \boldsymbol{t}(t)\|$, then we have $\langle\boldsymbol{n}, \boldsymbol{n}\rangle=1$ and $\langle\boldsymbol{n}, \boldsymbol{t}\rangle=0$. We can obtain a second smooth family of unit vector $\boldsymbol{b}(t)=\boldsymbol{t}(t) \wedge \boldsymbol{n}(t)$ normal to $\gamma$ at $t$. We remark that the triple $t(t), \boldsymbol{n}(t), \boldsymbol{b}(t)$ is the Lorentzian frame along $\gamma$. We now use the pseudoperpendicular lines spanned by $t(t)$, $\boldsymbol{n}(t), \boldsymbol{b}(t)$ as axes at $\gamma(t)$ with the unit points on the axes corresponding to the three given vectors. We remark that the Minkowski metric is invariant under the Lorentz transformation. Note the curve $\gamma(t)$ not necessarily unit speed, with $\gamma\left(t_{0}\right)=0$. Then the coordinates $\eta, \zeta$ and $\chi$ of $\gamma(t)$ relative to axes $\boldsymbol{t}, \boldsymbol{n}$ and $\boldsymbol{b}$ are functions of $t: \zeta(t)=\gamma(t) \cdot \boldsymbol{t}\left(t_{0}\right)$, $\eta(t)=\gamma(t) \cdot \boldsymbol{n}\left(t_{0}\right), \chi(t)=\gamma(t) \cdot \boldsymbol{b}\left(t_{0}\right), \eta(t)=f(\zeta(t)), \chi(t)=g(\zeta(t))$ where $f=f_{0}, g=g_{0}$. So $j^{k} f_{t}(0)=a_{2}(t) \zeta^{2}+a_{3}(t) \zeta^{3}+\cdots+a_{k}(t) \zeta^{k}, j^{k} g_{t}(0)=b_{2}(t) \zeta^{2}+b_{3}(t) \zeta^{3}+\cdots+b_{k}(t) \zeta^{k}$ in the neighbourhood $(0,0,0)$. Locally then $\gamma(I)$ can be written in the form $\left\{\zeta,\left(f_{t}(\zeta), g_{t}(\zeta)\right)\right\}$, with $f(0)=g(0)=j^{1} f_{t}(0)=j^{1} g_{t}(0)=0$. If $V_{k}$ denotes the space of polynomials in $\zeta$ of degree $\geqq 2$ and $\leqq k$ we have a map, the Lorentzian Monge-Taylor map for the space curve $\gamma, \mu_{\gamma}: I \rightarrow V_{k} \times V_{k}$ given by $\mu_{\gamma}(t)=\left(j^{k} f_{t}(0), j^{k} g_{t}(0)\right) .\left(V_{k} \times V_{k}\right.$ can be identified with $\mathbf{R}^{k-1} \times \mathbf{R}^{k-1}=\mathbf{R}^{2(k-1)}$ via the coordinates $\left(a_{2}, \cdots, a_{k}, b_{2}, \cdots, b_{k}\right)$.) Of course $\mu_{\gamma}$ depends rather heavily on our choice of unit normals $\boldsymbol{n}(t)$. Where, $a_{i}(t)=f_{i}(0)^{(i)} /(i !)$, $b_{i}(t)=g_{t}(0)^{(i)} /(i !)(2 \leqq i \leqq k)$, that is

$$
V_{k} \times V_{k}=\left\{\left(a_{2} \zeta^{2}+b_{3} \zeta^{3}+\cdots+b_{k} \zeta^{k}\right),\left(b_{2} \zeta^{2}+b_{3} \zeta^{3}+\cdots+a_{k} \zeta^{k}\right)\right\}
$$

Let $P_{k}$ denote the set of maps $\psi: \mathbf{R}_{1}^{3} \rightarrow \mathbf{R}_{1}^{3}$ of the form $\psi(x, y, z)=\left(\psi_{1}(x, y, z)\right.$, $\left.\psi_{2}(x, y, z), \psi_{3}(x, y, z)\right)$ where $\psi_{i}(x, y, z)$ is a polynomial in $x, y$ and $z$ of degree $\leq k$. So an element $\psi \in P_{k}$ is determined by the coefficients of the various monomials $x^{l} y^{i} z^{j}$ occurring in $\psi_{1}, \psi_{2}$ and $\psi_{3}$. There are altogether $1+3+\cdots+(2 k-1)+2 k+1=(k+1)^{2}$ monomials of degree $\leqq k$, so $P_{k}$ can be thought of as a Euclidean space $\mathbf{R}^{(k+1)^{2}}$. It is this space which will provide the required deformations of the curve.

To simplify matters we now assume that the curve $\gamma$ is proper (i.e. $\gamma$ satisfies the condition that the inverse image of a compact set is compact) and $\gamma(I)$ is bounded. The identity map $1_{\mathbf{R}_{1}^{3}}: \mathbf{R}_{1}^{3} \rightarrow \mathbf{R}_{1}^{3}$, is of course an element of $P_{k}$ (provided $k \geq 1$ ), and using the above assumption of $\gamma$ it easily follows that there is an open neighbourhood $U$ of $1_{\mathbf{R}_{1}^{3}}$ in $P_{k}$ with the property that if $\psi \in U$ then the linear map $T \psi(\gamma(t)): \mathbf{R}_{1}^{3} \rightarrow \mathbf{R}_{1}^{3} ; v \mapsto D \psi(\gamma(t)) \cdot v$ satisfy $D \psi(\gamma(t)) \cdot \boldsymbol{n}(t)$ is spacelike vector and $D \psi(\gamma(t)) \cdot \boldsymbol{t}(t)$ is timelike vector. Where, $D \psi(\gamma(t))$ denotes the derivative of $\psi$ at $\gamma(t)$. (In fact, in this case the two conditions can be written two conditions of algebraic inequality in the open neighbourhood of $\gamma(t)$, so by the compactness of $\gamma\left(S^{1}\right)$ the set which satisfy the two conditions is intersection of finite sets. Hence, it is open.) If we deform the original curve by the map $\psi$, then we can also obtain the required new smooth family of normal vectors $\boldsymbol{n}_{\psi}(t)$ as follows: since the map $\psi: \mathbf{R}_{1}^{3} \rightarrow \mathbf{R}_{1}^{3}$ 
is a diffeomorphism on some open set containing $\gamma(I)$, the vector $n(t)$ will be sent to some new spacelike vector $D \psi(\gamma(t)) n(t)$ which will be neither zero nor tangent to $\psi \circ \gamma$ at $t$. Orthogonally projecting this vector onto the normal plane to $\psi \circ \gamma$ at $t$ and normalizing, that is $\boldsymbol{n}_{\psi}(t)=\left(D \psi(\gamma(t)) \boldsymbol{n}(t)+\left\langle D \psi(\gamma(t)) \boldsymbol{n}(t), \boldsymbol{t}_{\psi}\right\rangle \boldsymbol{t}_{\psi}\right) /\left\|D \psi(\gamma(t)) \boldsymbol{n}(t)+\left\langle D \psi(\gamma(t)) \boldsymbol{n}(t), \boldsymbol{t}_{\psi}\right\rangle \boldsymbol{t}_{\psi}\right\|$, $\left\langle\boldsymbol{n}_{\psi}(t), \boldsymbol{n}_{\psi}(t)\right\rangle=1$. Where, $\boldsymbol{t}_{\psi}$ denotes the tangent vector of the curve $\psi \circ \gamma$ at $t$. So assuming as the above, we choose an open neighbourhood $U$ of $1_{I} \in P_{k}$ consisting of polynomial maps which map an open set containing $\gamma(I)$ diffeomorphically to its image. We have now shown that there is a smooth map

$$
\mu: I \times U \rightarrow V_{k} \times V_{k}
$$

defined by $\mu(-, \psi)=$ Monge-Taylor map for the curve $\psi \circ \gamma$ using the family of normal vectors $n_{\psi}(t)$. By exactly the same arguments as those in the proof of Theorem 9.9 in [4], we have the following theorem.

THEOREM 5.1. Let $\gamma$ be a nonlightlike curve and $Q$ a manifold in $V_{k} \times V_{k}=\mathbf{R}_{1}^{2 k-2}$. For some open set $U_{1} \subset U$ containing the identity map, the map $\mu: I \times U_{1} \rightarrow V_{k}$ defined by $\mu(t, \psi)=\mu_{\psi \circ \gamma}(t)$ is transverse to $Q$. (In fact we can prove that $\mu$ is a submersion so $Q$ does not enter the argument at all.)

In order to give a proof of Theorem A, we prepare some lemmas.

LEMMA 5.2. (1) Let $\gamma: I \rightarrow \mathbf{R}_{1}^{3}$ be a timelike curve defined by $\gamma(t)=\left(\zeta, f_{t}(\zeta)\right.$, $\left.g_{t}(\zeta)\right)=\left(a_{2} \zeta^{2}+a_{3} \zeta^{3}+\cdots, \zeta, b_{2} \zeta^{2}+b_{3} \zeta^{3}+\cdots\right)$ with $\zeta\left(t_{0}\right)=0$ and $k\left(s_{0}\right) \neq 0$. We denote that $M_{i j}=a_{i} b_{j}-a_{j} b_{i}, N_{i j}=a_{i} a_{j}-\varepsilon(\gamma) b_{i} b_{j}$. Then:

(a) $\tau\left(s_{0}\right)=\tau^{\prime}\left(s_{0}\right)=0$ at $t_{0}$ if and only if

$$
\left\{\begin{array}{l}
M_{32}=0 \\
2 M_{42} N_{22}-3 M_{32} N_{23}=0 .
\end{array}\right.
$$

(b) $\left(k k^{\prime} \tau^{\prime}-k k^{\prime \prime} \tau+k^{2} \tau^{3}+2 k^{2} \tau\right)\left(s_{0}\right)=\left(k k^{\prime} \tau^{\prime}-k k^{\prime \prime} \tau+k^{2} \tau^{3}+2 k^{\prime 2} \tau\right)^{\prime}\left(s_{0}\right)=0$ at $t_{0}$ with $k^{\prime}\left(s_{0}\right) \neq 0$ and $\tau\left(s_{0}\right) \neq 0$, if and only if

$$
\left\{\begin{aligned}
A_{1}=4 M_{42} N_{23} N_{22}+9 M_{23} N_{23}^{3}-4 M_{32} N_{24} N_{22}-3 M_{32} N_{33} N_{22}-4 M_{32} N_{22}^{3}+3 M_{32}^{3}=0 \\
A_{2}=N_{23}\left(18 M_{32}^{3}+12 M_{43} N_{22}^{2}+20 M_{52} N_{22}^{2}+48 M_{24} N_{23} N_{22}-72 M_{23} N_{23}^{2}\right. \\
\left.\quad+24 M_{23} N_{24} N_{22}+18 M_{23} N_{22} N_{33}\right)-M_{32}\left(27 N_{23}^{3}+72 N_{23} N_{22}^{3}-36 N_{23} N_{24} N_{22}\right. \\
\left.\quad-27 N_{23} N_{22} N_{33}+36 N_{34} N_{22}^{2}+20 N_{25} N_{22}^{2}+3 N_{22}^{2} N_{33}\right)+3\left(M_{32}^{2}+N_{23}^{2}\right)\left(12 M_{42} N_{22}\right. \\
\left.+18 M_{23} N_{23}\right)+3 M_{32} N_{23}\left(9 N_{23}^{2}+12 N_{24} N_{22}+9 N_{22} N_{33}+12 N_{22}^{3}\right)=0 .
\end{aligned}\right.
$$

(2) Let $\gamma: I \rightarrow \mathbf{R}_{1}^{3}$ be a spacelike curve defined by $\gamma(t)=\left(f_{t}(\zeta), \zeta, g_{t}(\zeta)\right)=$ $\left(a_{2} \zeta^{2}+a_{3} \zeta^{3}+\cdots, \zeta, b_{2} \zeta^{2}+b_{3} \zeta^{3}+\cdots\right)$ with $\zeta\left(t_{0}\right)=0$ and $k\left(s_{0}\right) \neq 0$. And we denote that $M_{i j}=a_{i} b_{j}-a_{j} b_{i}, N_{i j}=a_{i} a_{j}-\varepsilon(\gamma) b_{i} b_{j}$.

Then:

(a) $\tau=0$ and $\tau^{\prime}=0$ at to if and only if

$$
\left\{\begin{array}{l}
M_{32}=0 \\
2 M_{42}\left|N_{22}\right|+3 M_{32} N_{32}=0 .
\end{array}\right.
$$


(b) By the direct calculation, $\left(k k^{\prime} \tau^{\prime}-k k^{\prime \prime} \tau-k^{2} \tau^{3}+2 k^{\prime 2} \tau\right)\left(s_{0}\right)=\left(2 k k^{\prime} \tau^{3}-k k^{\prime} \tau^{\prime \prime}+\right.$ $\left.k k^{(3)} \tau+3 k^{2} \tau^{2} \tau^{\prime}-3 k^{\prime} k^{\prime \prime} \tau-3 k^{\prime 2} \tau^{\prime}\right)\left(s_{0}\right)=0$ at $t_{0}$ with $k^{\prime}\left(s_{0}\right) \neq 0$ and $\tau\left(s_{0}\right) \neq 0$ if and only if

$$
\left\{\begin{array}{l}
B_{1}= \pm 4 M_{42} N_{23}\left|N_{22}\right|+3 M_{23} N_{23}^{2} \mp 4 M_{32} N_{24}\left|N_{22}\right| \mp 3 M_{32} N_{33}\left|N_{22}\right|+4 M_{32}\left|N_{22}\right|^{2} N_{22} \\
\quad-3 M_{32}^{3}=0 \\
B_{2}= \pm N_{23}\left(18 M_{32}^{3}+12 M_{42}\left|N_{22}\right|^{2}+20 M_{52}\left|N_{22}\right|^{2} \pm 48 M_{24} N_{23}\left|N_{22}\right|-72 M_{23} N_{23}^{2}\right. \\
\left.\quad \pm 24 M_{23} N_{24}\left|N_{22}\right| \pm 18 M_{23}\left|N_{22}\right| N_{33}\right)+M_{32}\left(27 N_{23}^{3}+72 N_{23} N_{22}\left|N_{22}\right|^{2}\right. \\
\left.\quad-36 N_{23} N_{24}\left|N_{22}\right|-27 N_{23}\left|N_{22}\right| N_{33} \pm 36 N_{34}\left|N_{22}\right|^{2} \pm 20 N_{25}\left|N_{22}\right|^{2} \pm 3\left|N_{22}\right|^{2} N_{33}\right) \\
\quad-3 M_{32} N_{23}\left(-9 N_{23}^{2}+12 N_{24}\left|N_{22}\right|+9\left|N_{22}\right| N_{33}-12 N_{22}\left|N_{22}\right|^{2}\right) \\
\quad-3\left(M_{32}^{2}-N_{23}^{2}\right)\left(12 M_{42}\left|N_{22}\right| \pm 18 M_{23} N_{23}\right)=0
\end{array}\right.
$$

Here $\zeta$ is the coordinate along the $t$-direction, $f_{t}(\zeta)$ along the $n$-direction and $g_{t}(\zeta)$ along the $b$-direction.

Since the proof of Lemma 5.2 is analogous to that of Lemma 5.2 in [6], we omit it.

LEMMA 5.3. We consider smooth maps $\rho_{i}: V_{4} \times V_{4}=\mathbf{R}^{6} \rightarrow \mathbf{R}(i=1,2)$ given by

$$
\left\{\begin{array}{l}
\rho_{1}=M_{32} \\
\rho_{2}=2 M_{42} N_{22}-3 M_{32} N_{23}
\end{array}\right.
$$

$\theta_{i}: V_{4} \times V_{4}=\mathbf{R}^{6} \rightarrow \mathbf{R}(i=1,2)$ given by

$$
\left\{\begin{array}{l}
\theta_{1}=M_{32} \\
\theta_{2}=2 M_{42}\left|N_{22}\right|+3 M_{32} N_{32}
\end{array}\right.
$$

$\phi_{i}: V_{5} \times V_{5}=\mathbf{R}^{8} \rightarrow \mathbf{R}(i=1,2)$ given by

$$
\left\{\begin{array}{l}
\phi_{1}=A_{1} \\
\phi_{2}=A_{2}
\end{array}\right.
$$

and $\mu_{i}: V_{5} \times V_{5}=\mathbf{R}^{8} \rightarrow \mathbf{R}(i=1,2)$ given by

$$
\left\{\begin{array}{l}
\mu_{1}=B_{1} \\
\mu_{2}=B_{2}
\end{array} .\right.
$$

Here, $M_{i j}, N_{i j}, P_{i j}, A_{i}$ and $B_{i}$ are given as the above. Then:

(1) The set $Q_{1}=\left\{\left(a_{2}, a_{3}, a_{4}, b_{2}, b_{3}, b_{4}\right) \in \mathbf{R}^{6} \mid \rho_{1}=\rho_{2}=0\right\}$ is a codimension two submanifold in $\mathbf{R}^{8}$.

(2) The set $Q_{2}=\left\{\left(a_{2}, a_{3}, a_{4}, b_{2}, b_{3}, b_{4}\right) \in \mathbf{R}^{6} \mid \theta_{1}=\theta_{2}=0\right\}$ is a codimension two submanifold in $\mathbf{R}^{8}$.

(3) The set $Q_{3}=\left\{\left(a_{2}, a_{3}, a_{4}, a_{5}, b_{2}, b_{3}, b_{4}, b_{5}\right) \in \mathbf{R}^{8} \mid \phi_{1}=\phi_{2}=0\right\}$ is a codimension two submanifold in $\mathbf{R}^{8}$.

(4) The set $Q_{4}=\left\{\left(a_{2}, a_{3}, a_{4}, a_{5}, b_{2}, b_{3}, b_{4}, b_{5}\right) \in \mathbf{R}^{8} \mid \mu_{1}=\mu_{2}=0\right\}$ is a codimension two submanifold in $\mathbf{R}^{8}$. 
PROOF. (1) The Jacobian matrix of the map $\left(\rho_{1}, \rho_{2}\right)$ is calculated as follows:

$$
J\left(\rho_{1}, \rho_{2}\right)=\left(\begin{array}{cccccc}
\frac{\partial \rho_{1}}{\partial a_{2}} & b_{2} & 0 & \frac{\partial \rho_{1}}{\partial b_{2}} & -a_{2} & 0 \\
\frac{\partial \rho_{2}}{\partial a_{2}} & \frac{\partial \rho_{2}}{\partial a_{3}} & 2 b_{2}\left(\frac{k}{2}\right)^{2} & \frac{\partial \rho_{2}}{\partial b_{2}} & \frac{\partial \rho_{2}}{\partial b_{3}} & -2 a_{2}\left(\frac{k}{2}\right)^{2}
\end{array}\right)
$$

Since $k\left(s_{0}\right)=2\left(a_{2}{ }^{2}+b_{2}{ }^{2}\right)^{1 / 2} \neq 0$, the numbers $a_{2}$ and $b_{2}$ are not both zero. That is rank $J\left(\rho_{1}, \rho_{2}\right)=2$. This means that $Q_{1}$ is a submanifold in $\mathbf{R}^{8}$ with codimension two.

(4) The Jacobian matrix of the map $\left(\mu_{1}, \mu_{2}\right)$ is also calculated as follows:

$J\left(\mu_{1}, \mu_{2}\right)=$

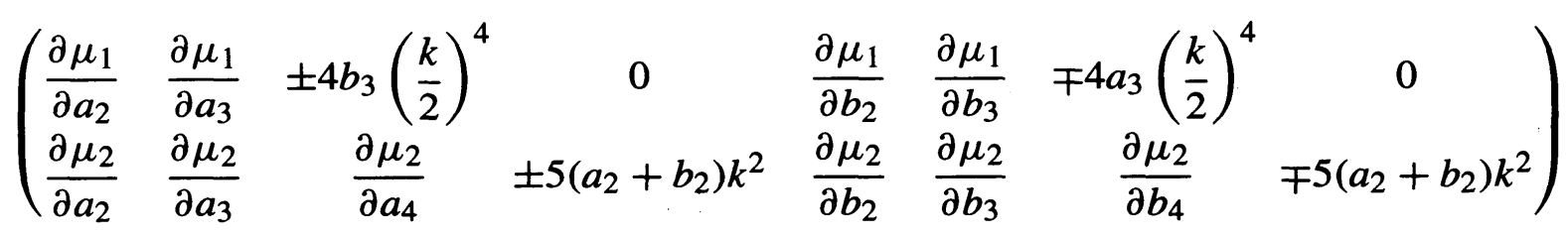

where $k=2\left|a_{2}{ }^{2}-b_{2}{ }^{2}\right|^{1 / 2} \neq 0$. Then $b_{2} \pm a_{2} \neq 0$. And either $a_{3} \neq 0$ or $b_{3} \neq 0$ by $\tau\left(s_{0}\right) \neq 0$. Hence rank $J\left(\phi_{1}, \phi_{2}\right)=2$. So that $Q_{4}$ is a submanifold in $\mathbf{R}^{8}$. with codimension two.

The assertions (2) and (3) follows similar arguments as in the proofs of cases (1) and (4). This completes the proof.

We can use Theorem 5.1, Lemmas 5.2 and 5.3 for the proof of Theorem A exactly the same way as the proof of Corollary 9.7 in [4]. So we omit the detail here.

ACKnowledgment. We would like to thank Professor S. Izumiya for proposing the problems in this paper and for helpful advice.

\section{References}

[ 1 ] J. W. BRUCE, On singularities, envelopes and elementary differential geometry, Math. Proc. Cambridge Philos. Soc. 89 (1981), 43-48.

[ 2 ] J. W. BRUCE and P. J. Giblin, Generic curves and surfaces, J. London Math. Soc. 24 (1981), 555-561.

[ 3 ] J. W. BrUCE and P. J. Giblin, Generic Geometry, Amer. Math. Monthly 90 (1983), 529-545.

[4] J. W. BRUCE and P. J. GiBlin, Curves and singularities (second edition), Cambridge Univ. Press (1992).

[ 5 ] D. L. FIDAL and P. J. Giblin, Generic 1-parameter families of caustics by reflexion in the plane, Math. Proc. Camb. Phil. Soc. 96 (1984), 425-432.

[6] S. IzUmiYA, D. PEI and T. SANo, The lightcone Gauss map and the lightcone developable of a spacelike curve in Minkowski 3-space, Hokkaido University preprint series in Math. 411 (1998).

[ 7 ] S. IzUmiYA and A. TAKiYAmA, A time-like surface in Minkowski 3-space which contains pseudocircles, Proc. Edinburgh Math. Soc. 40 (1997), 127-136.

[ 8 ] M. KossowsKI, The Null Blow-Up of a Surface in Minkowski 3-space and Intersection in the Spacelike Grassman, Michigan Math. J. 38 (1991), 401-415.

[ 9 ] D. K. H. MochidA, R. C. Romero-Fuster and M. A. RuAS, The geometry of surfaces in 4-space from a contact viewpoint, Geom. Dedicata 54 (1995), 323-332.

[10] B. O’ Neill, Semi-Riemannian Geometry, Academic Press, New York (1983). 
[11] D. PEI, Singularities of $\mathbf{R} P^{2}$-valued Gauss maps of surfaces in Minkowski 3-space, to appear in Hokkaido Math. J.

[12] I. PORTEOUS, The normal singularities of submanifold, J. Differential Geom. 5 (1971), 543-564.

\section{Present Addresses:}

DONGHE PEI

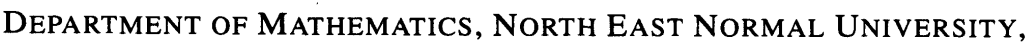
Chang Chun 130024, P.R. China.

TAKASHI SANO

FACULTY OF ENGINEERING,

HOKKAI-GAKUEN UNIVERSITY, SAPPORO 062-8605, JAPAN. 